\title{
Nanoscale
}

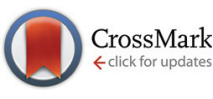

Cite this: Nanoscale, 2015, 7, 5140

Received 14th January 2015, Accepted 12th February 2015

DOI: $10.1039 / c 5 n r 00295 h$

www.rsc.org/nanoscale

\section{Cucurbit[n]uril-capped upconversion nanoparticles as highly emissive scaffolds for energy acceptors $\uparrow$}

\author{
Laura Francés-Soriano, María González-Béjar* and Julia Pérez-Prieto*
}

\begin{abstract}
Spontaneous adsorption of cucurbit $[n]$ uril $\mathrm{CB}[n](n=6,7$, and 8 ) on the surface of naked upconversion nanoparticles (UCNPs), in particular, $\mathrm{NaYF}_{4}: \mathrm{Er}^{3+}(2 \%), \mathrm{Yb}^{3+}(18 \%)$ gave rise to UCNP@CB[n] exclusion complexes. These complexes proved to be highly stable as well as highly emissive under near-infrared excitation. By using two tricyclic basic dyes (specifically, methylene blue and pyronin $Y)$ as a proof of concept, we demonstrate that the UCNP@CB[n] $(n=6,7)$ nanohybrids can form exclusion complexes with this type of dyes via the CB carbonyl free portal, i.e., UCNPaCBadye hybrids, thus making it possible to locate a high concentration of the dyes close to the UCNP and, consequently, leading to efficient energy transfer from the UCNP to the dye.
\end{abstract}

Upconversion nanoparticles (UCNPs), such as ytterbium and erbium co-doped sodium yttrium fluoride nanoparticles $\left(\mathrm{NaYF}_{4}: \mathrm{Er}^{3+}, \mathrm{Yb}^{3+}\right)$, emit in the visible after being excited in the near infrared (NIR) due to their intra-configurational $4 \mathrm{f}^{n}$ electron transitions. ${ }^{1,2}$ They display unique photoluminescence properties, such as anti-Stokes luminescence, narrow emission bands and good chemical stability. In addition, the absence of autofluorescence, photobleaching and photoblinking together with the deep tissue penetration of the NIR light make them suitable for bioapplications. ${ }^{2-8}$

UCNPs are usually synthesised at high temperatures by using organic capping ligands and non-coordinating solvents (e.g. oleic acid and 1-octadecene). ${ }^{9-13}$ Their poor water-dispersibility is the main handicap for their bioapplication and their derivatisation with polymers or silica shell is being extensively studied. ${ }^{14-16}$ Another strategy to make them water dispersible is by making them naked (i.e. free of ligand $)^{17}$ or by encapsulating the lipophilic ligands of the UCNPs inside the cavity of macromolecules like cyclodextrins. ${ }^{5,18,19}$

Instituto de Ciencia Molecular (ICMol)/Departamento de Química Orgánica, Universidad de Valencia, C/Catedrático José Beltrán 2, 46980 Paterna, Valencia, Spain.E-mail: julia.perez@uv.es, maria.gonzalez@uv.es; Fax: +34-963543576; Tel: $+34-963543050$

$\dagger$ Electronic supplementary information (ESI) available: Experimental methods, TEM images, EDX, XRD, absorption and emission spectra, ${ }^{1} \mathrm{H}-\mathrm{NMR}$. See DOI: $10.1039 / \mathrm{c} 5 \mathrm{nr} 00295 \mathrm{~h}$
Cucurbit $[n]$ urils (CB $[n], n=5-10)$ are another kind of watersoluble macrocyclic hosts with considerable molecular recognition abilities..$^{20,21}$ They present a "pumpkin" shape with the urea oxygen atoms at the edges and tilted inwards and have a height of $0.91 \mathrm{~nm} .^{22}$ They form strong inclusion complexes with many types of organic guests by making use of their internal hydrophobic cavity. ${ }^{20}$ Interestingly, the carbonylfringed portals of $\mathrm{CB}[n]$ can interact with metallic cations ${ }^{23-25}$ and bind to gold surfaces, ${ }^{26}$ as well as to the surface of silver, ${ }^{27,28}$ platinum, ${ }^{29}$ palladium, ${ }^{30}$ and gold nanoparticles. ${ }^{31-33}$ Furthermore, the interaction between $\mathrm{CB}[n]$ and lanthanide cations has also been demonstrated. ${ }^{34-38}$

With regard to $\mathrm{CB}$ guests, the host-guest complexes between $\mathrm{CB}[n]$ and tricyclic basic dyes have been studied previously and depending on the $\mathrm{CB}$ size, $1: 1$ or $1: 2$ complexes can be formed. ${ }^{39,40}$ For example, $\mathrm{CB}[7]$ encapsulates a methylene blue (MB) monomer, while an $\mathrm{MB}$ dimer $\left(\mathrm{MB}_{2}\right)$ is encapsulated inside $\mathrm{CB}[8] .^{39}$ Encapsulation of dyes inside the $\mathrm{CB}[n]$ cavities can be advantageous: enhanced fluorescence emission and lifetime, photostabilisation, increased solubility in water, and de-aggregation. ${ }^{39}$ Tricyclic basic dyes have been extensively studied as dye lasers and photosensitizers, among other applications. ${ }^{41}$

Regarding the formation of $\mathrm{CB}$ exclusion complexes, we have recently prepared $\mathrm{CB}[7]$-capped AuNPs (Au@CB) by reacting naked AuNPs with $\mathrm{CB}[7]$ and we have demonstrated that $\mathrm{Na}^{+}$and $\mathrm{NH}_{4}{ }^{+}$ions block the entrance of $\mathrm{O}_{2}$ to the $\mathrm{CB}$ cavity of the Au@CB hybrids. ${ }^{31}$

Now, we have focused on the potential assembling of CBs on the naked UCNPs to obtain UCNP@CB nanohybrids. The $\mathrm{CB}$ units could be exploited as hosts for the functional molecules (FM), thus placing them close to the UCNP surface. Alternatively, the CB cavity could host a moiety attached to the FM, thus placing the FM at a certain distance from the UCNP surface. In addition, the UCNP@CB could be used as a scaffold for FM to build an UCNP@CB@FM exclusion complex by making use of a favourable interaction between the molecule and the CB free carbonyl portal. In the last case, the UCNP@CB nanohybrids would allow the increase of the local concentration 


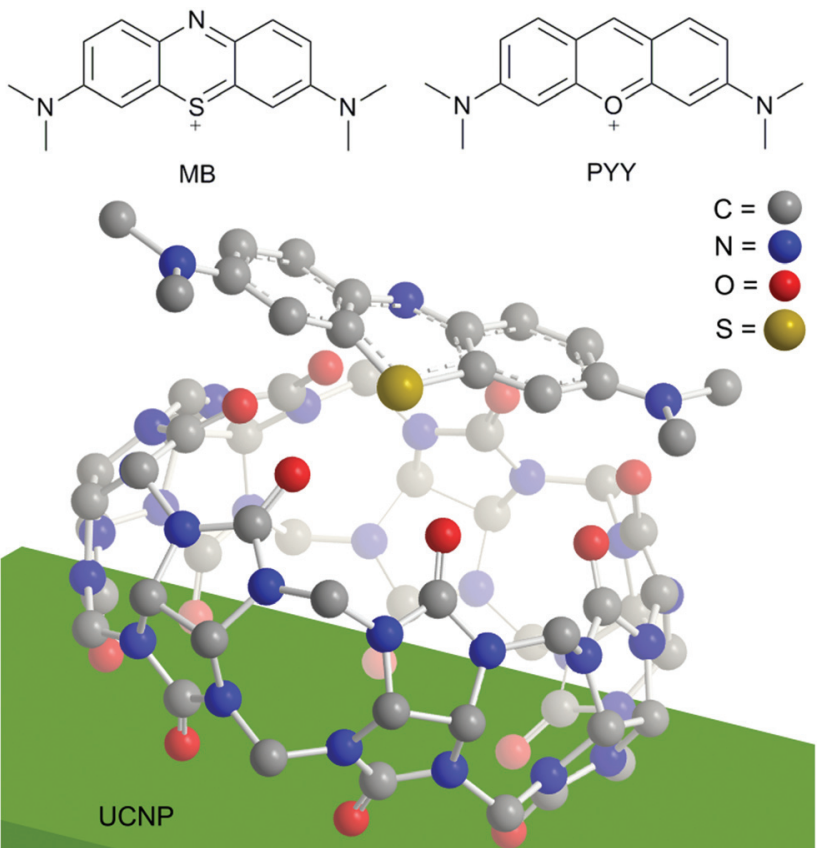

Fig. 1 Top: Structure of the dyes used in this study, methylene blue (MB) and pyronin Y (PYY). Bottom: Proposed interaction between the UCNP, cucurbit[7]uril (CB[7]), and MB. The outer diameter of $\mathrm{CB}[7]$ is $1.6 \mathrm{~nm}^{22}$ while the MB long axis is $1.275 \mathrm{~nm}^{42}$

of a FM close to the UCNP surface while fixing their relative orientation and it would be more accessible to other molecules as if they were encapsulated inside the CB cavity.

In this report, we demonstrate that the naked UCNPs form stable UCNP@CB exclusion complexes with different sizedCBs and the UCNP@CB nanohybrids can be used as scaffolds as well as energy donors for cationic dyes anchored to the $\mathrm{CB}$ free carbonyl portal (a charge-dipole interaction). We have used MB and pyronin Y (PYY) as a proof of concept (see Fig. 1).

\section{Preparation of UCNP@CB nanohybrids}

Naked $\mathrm{NaYF}_{4}: \mathrm{Yb}^{3+}(18 \%), \mathrm{Er}^{3+}(2 \%)$ UCNPs were synthesised in two steps. First, oleate-capped nanoparticles (UCNP@OA) were prepared by following a previously described protocol ${ }^{43}$ and then, the oleate ligand was removed by acidification with HCl. ${ }^{17}$ UCNP@CB nanohybrids were prepared by sonication of a mixture of the corresponding $\mathrm{CB}[n](n=6,7,8)$ and the naked UCNP in water for $15 \mathrm{~min}$, followed by stirring the mixture at room temperature for $24 \mathrm{~h}$. Centrifugation made the separation of the UCNP (white powder) from the $\mathrm{CB}[n]$ excess possible (see experimental details in ESI $\dagger$ ).

The nanoparticles were characterised by transmission electron microscopy (TEM and high resolution TEM, HRTEM). As shown in Fig. 2 and $\mathrm{S} 1, \uparrow$ the naked UCNPs were uniform hexagonal prisms and their height and length were $(32.7 \pm 1.7)$ and $(19.1 \pm 1.4) \mathrm{nm}$, respectively. The addition of $\mathrm{CB}[n]$ to the naked UCNPs did not change their shape or size (Fig. 2).

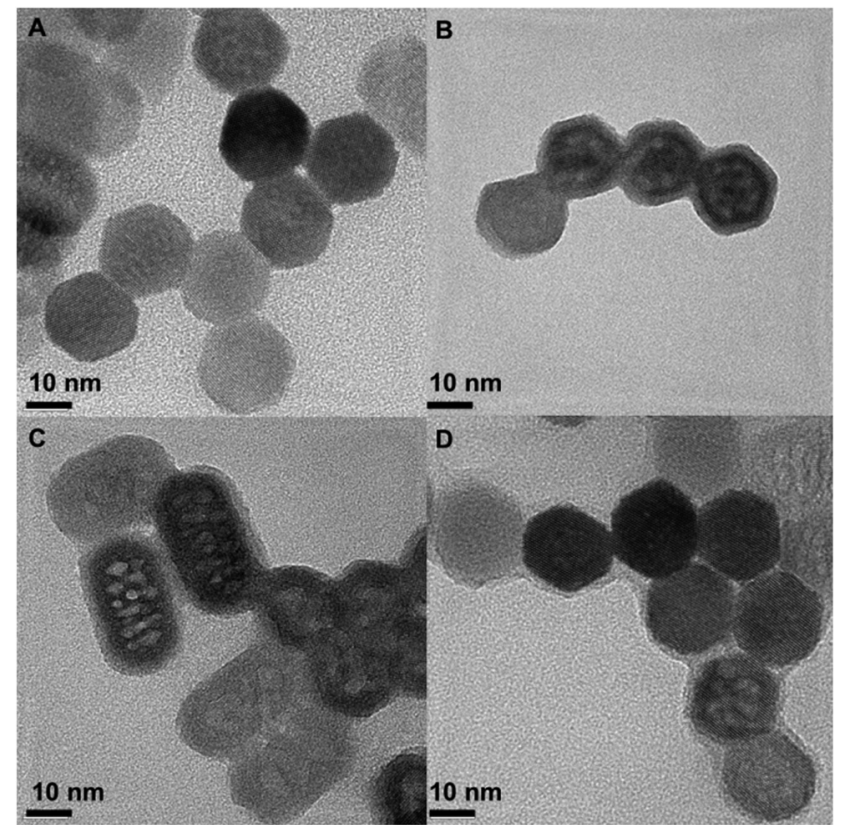

Fig. 2 Representative HRTEM image of the naked UCNPs (A), UCNP@CB[6] (B), UCNP@CB[7] (C), and UCNP@CB[8] (D).

HRTEM images show the thin organic capping of the UCNP@CB $[n]$ nanohybrids. The thickness of this capping was $1.2 \pm 0.1 \mathrm{~nm}$ in the case of UCNP@CB[6], $1.5 \pm 0.3 \mathrm{~nm}$ in the UCNP@CB[7], and $1.2 \pm 0.2$ for the UCNP@CB[8]. These data are consistent with the height of the $\mathrm{CB}[n](9.1 \AA) .{ }^{44}$

The energy-dispersive X-ray (EDX) analysis and X-ray diffraction (XRD) spectrum of the UCNPs corroborated the hexagonal phase of the $\mathrm{NaYF}_{4}: \mathrm{Er}^{3+}(2 \%), \mathrm{Yb}^{3+}(18 \%)$ nanoparticles; see Fig. S2 and S3.†

Fourier transform infrared (FTIR) spectroscopy was carried out to verify that the UCNPs were capped with the different $\mathrm{CB}[n]$ via one of the carbonyl portals. The FTIR spectrum of $\mathrm{CB}[6], \mathrm{CB}[7], \mathrm{CB}[8]$, and the respective CB-capped UCNPs are shown in Fig. 3. The strong band assigned to the $\mathrm{CB} \nu_{\mathrm{as}}(\mathrm{C}=\mathrm{O})$ stretching vibration shifted to higher values in the nanohybrid. The shift was greater in the case of UCNP@CB[6] and UCNP@CB[7] $\left(15 \mathrm{~cm}^{-1}\right.$ and $17 \mathrm{~cm}^{-1}$, respectively) than in UCNP@CB[8] $\left(4 \mathrm{~cm}^{-1}\right)$.

These changes corroborated the interaction of the CBs with the UCNP surface through the carbonyl groups (Fig. 1). The $\nu_{\text {as }}(\mathrm{C}=\mathrm{O})$ stretching at higher values suggests a further deviation from the planarity of the $\mathrm{C}=\mathrm{O}$ bond compared with the $\mathrm{N}-\mathrm{C}-\mathrm{N}$ plane when the $\mathrm{CB}$ anchored to the nanoparticle surface and this is significant for the smaller CBs. The smaller shift $\left(4 \mathrm{~cm}^{-1}\right)$ for the largest $\mathrm{CB}$ as compared to that for the smaller ones $\left(\sim 15-17 \mathrm{~cm}^{-1}\right)$ can be attributed to a less effective interaction of the $\mathrm{CB}$ with the nanoparticle surface (see below).

Emission spectra of the nanohybrids were compared with that of the naked UCNPs (Fig. 4). These spectra were recorded at a concentration of $1 \mathrm{mg}$ (nanoparticle core) in a $1 \mathrm{ml}$ solution, using front face excitation at $980 \mathrm{~nm}$. 


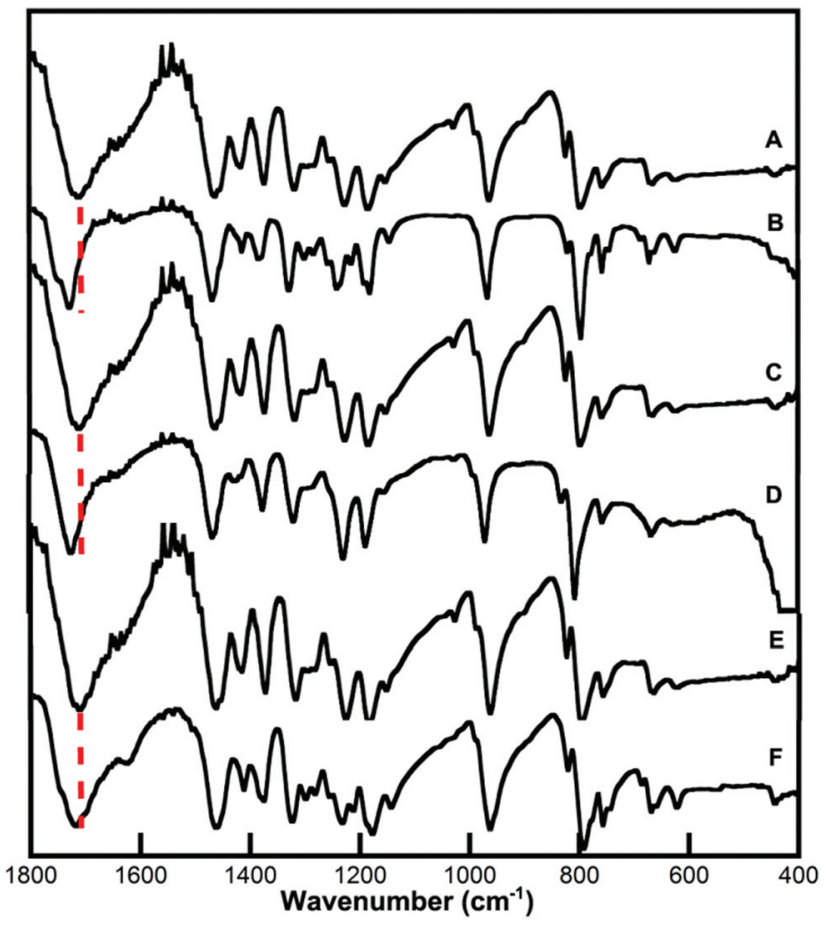

Fig. 3 FTIR spectra of (A) $\mathrm{CB}[6]$, (B) UCNP@CB[6], (C) $\mathrm{CB}[7]$, (D) UCNP@CB[7], (E) CB[8] and (F) UCNP@CB[8].

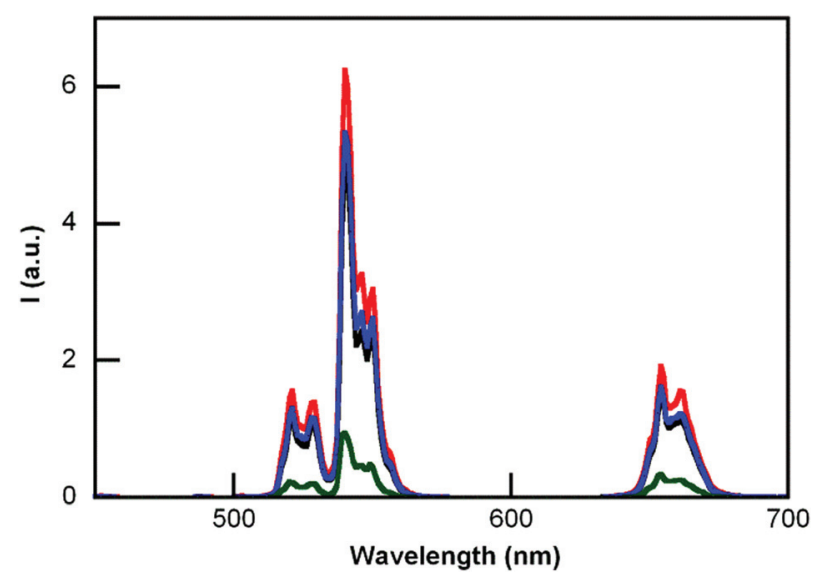

Fig. 4 Emission spectra $\left(\lambda_{\mathrm{ex}}=980 \mathrm{~nm}\right)$ of aqueous dispersions of UCNP@CB[6] (black line), UCNP@CB[7] (red line), UCNP@CB[8] (blue line) and the naked UCNP (green line).

The emission intensity of the naked UCNP drastically increased due to the CB-capping. The enhancement recorded at $540 \mathrm{~nm}$ was $4.9,6.9$, and 5.4 times more than that of the naked UCNP for UCNP@CB[6], UCNP@CB[7], and UCNP@CB[8], respectively. Comparatively, the emission of the UCNP@OA nanoparticles in chloroform was only 3.5 times greater than that of the naked UCNPs in water (Fig. S4 $\dagger$ ).

Thermogravimetric analyses (TGA) of the UCNP@CB nanohybrids and CBs were performed to gain insight into the $\mathrm{CB}[n]$ loading on the UCNP surface. The peak of the first derivative indicates the point of the greatest rate of change in the weight

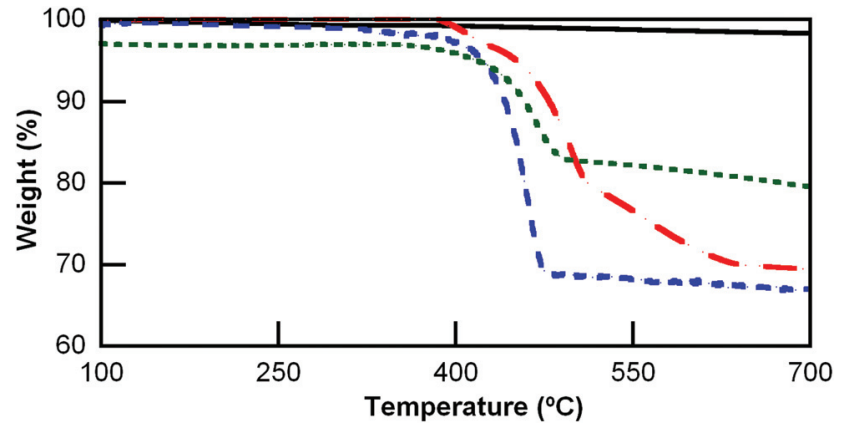

Fig. 5 Thermogravimetric analysis (TGA) of the UCNP (-), UCNP@CB [6] (—), UCNP@CB[7] (—) and UCNP@CB[8] (----).

loss curve. No decomposition was observed up to $420{ }^{\circ} \mathrm{C}$ for $n=6$ and 8, although $\mathrm{CB}[7]$ starts decomposing at a lower temperature $\left(370^{\circ} \mathrm{C}\right)$; this is in agreement with the differences detected for the different sized CBs. ${ }^{44}$ Fig. 5 compares the TGA spectrum of the naked UCNPs with that of UCNP@CB[7]. The weight loss before reaching $500{ }^{\circ} \mathrm{C}$ can mostly be attributed to the loss of $\mathrm{CB}[n]$ which is $31 \%, 33 \%$, and $14 \%$ for UCNP@CB[6], UCNP@CB[7], and UCNP@CB[8], respectively.

\section{UCNPACB nanohybrids as scaffolds and energy donors}

As proof of concept, the versatility of the nanohybrids as scaffolds and energy donors was tested with two cationic dyes, in particular methylene blue (MB) and pyronin Y (PYY).

Increasing concentrations of $\mathrm{MB}$ were added to water solutions of the UCNP@CB $[n]$ nanohybrids ( $2 \mathrm{~mL}$ of a $0.018 \mathrm{mg}$ $\mathrm{mL}^{-1}$ of UCNP@CB $[n]$ in water). According to the TGA data, the $\mathrm{CB}[n]$ molar concentration of the UCNP@CB $[n]$ dispersions was 5.5, 5.25, and $2.4 \mu \mathrm{M}$ for UCNP@CB[6], UCNP@CB[7], and UCNP@CB[8], respectively. Aliquots of aqueous $\mathrm{MB}$ solutions were added (up to $100 \mu \mathrm{L}$ ) until the $\mathrm{MB}$ and $\mathrm{CB}[n]$ molar concentrations were the same.

The emission spectra $\left(\lambda_{\mathrm{ex}}\right.$ at $\left.980 \mathrm{~nm}\right)$ were recorded after each addition. Fig. 6 shows the decrease of UCNP@CB[7] emission (see bands with $\lambda_{\max } c a$. $650 \mathrm{~nm}$ and $540 \mathrm{~nm}$ ) at the highest $\mathrm{MB}$ concentration. A similar trend was found for UCNP@CB[6], while MB led to insignificant quenching of the UCNP@CB[8] emission (Fig. S5 $\dagger$ ). The different trend in the emission quenching for the nanohybrids should be related to the type of interaction between the UCNP@CB $[n]$ nanohybrid and $\mathrm{MB}$, i.e., the formation of either an exclusion or inclusion complex and the hosting of either $\mathrm{MB}$ or $\mathrm{MB}_{2}$.

The spectra of the UCNP@CB[8]/MB mixtures showed the efficient formation of the $\mathrm{MB}_{2}$ dimer $^{39}$ with $\lambda_{\max }$ at $c a .611 \mathrm{~nm}$ and consequently the emission of these mixtures was practically non-existent (Fig. 7 and S6†).

Remarkably, the absorption and emission spectra of the UCNP@CB[7]/MB mixtures (at $\lambda_{\max }$ at ca. 664 and $684 \mathrm{~nm}$, 


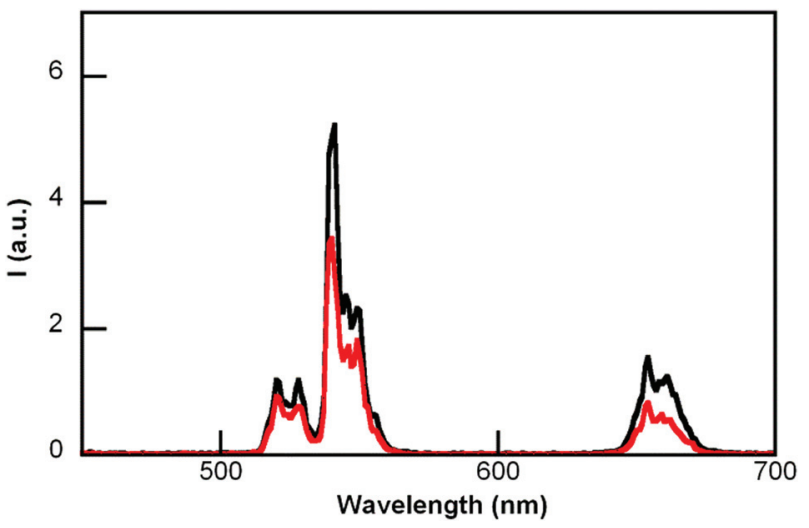

Fig. 6 Emission spectra ( $\lambda_{\text {ex }} 980 \mathrm{~nm}$ ) of UCNP@CB[7] before and after the addition of $M B$ ( $M B$ and $C B$ were at the same concentration).
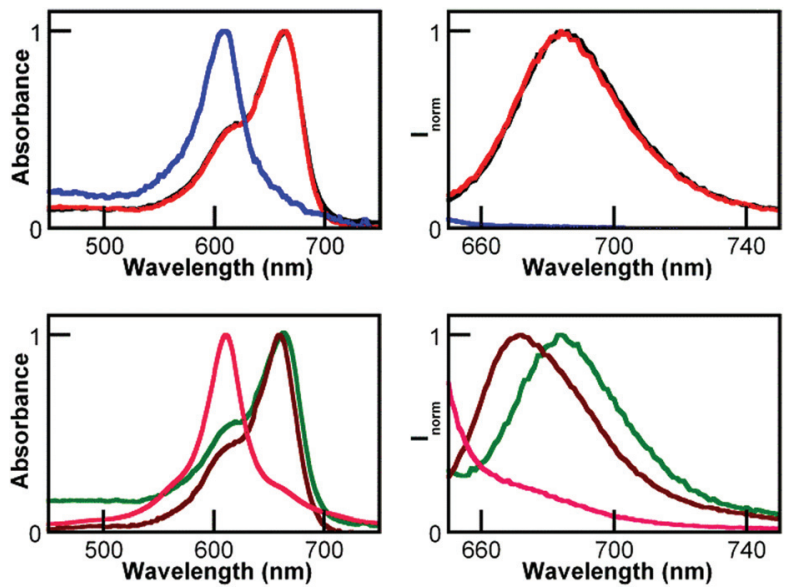

Fig. 7 Top: (left) Absorption and (right) emission spectra at $\lambda_{\text {exc }}=$ $640 \mathrm{~nm}$ of water solutions of UCNP@CB[n]/MB containing MB and CB at the same concentration: UCNP@CB[6]/MB (black line), UCNPaCB[7]/MB (red line), and UCNP@CB[8]/MB (blue line); the absorption spectra of the UCNPaCB[n]/MB mixture as well as the emission spectra of UCNP@CB[n]/MB ( $n=6$ and 7) have been normalized. Bottom: (left) Absorption and (right) emission spectra at $\lambda_{\text {exc }}=640 \mathrm{~nm}$ of water solutions of $\mathrm{CB}[6] / \mathrm{MB}$ (green line), $\mathrm{CB}[7] / \mathrm{MB}$ (brown line), and $\mathrm{CB}[8] / \mathrm{MB}$ (pink line); all of them at the same MB concentration. The spectra of $\mathrm{CB}[n] / \mathrm{MB}(n=6$ and 7$)$ have been normalized.

respectively) were coincident with that of UCNP@CB[6]/MB and free MB; see Fig. 7 left and S6. $\dagger$

It has to be taken into account that $\mathrm{CB}[7]$ can encapsulate MB and this results in a blue-shifted absorption and emission (Fig. 7) due to the formation of the $\mathrm{CB}[7] @ \mathrm{MB}$ inclusion complex, ${ }^{31}$ which is not possible for $\mathrm{CB}[6]$.

These results are consistent with the formation of exclusion complexes between the UCNP@CB $[n](n=6,7)$ nanohybrids and $\mathrm{MB}$, i.e., the formation of UCNP@CB $[n] @ M B$ nanosystems due to a more favourable interaction between the cationic dye and the CB free carbonyl portal of the UCNP@CB $[n]$ nanohybrid than between the CB cavity and the cationic dye (Fig. 1).

The ${ }^{1} \mathrm{H}$-NMR spectrum of the UCNP@CB[7]/MB (the same concentration of $\mathrm{CB}$ and $\mathrm{MB}$ ) was recorded to corroborate the anchoring of MB to the UCNP@CB nanohybrid and to gain insight into the orientation of $\mathrm{MB}$ in the nanosystem. As expected, the signals of the $\mathrm{CB}$ and $\mathrm{MB}$ moieties were broader in the UCNP@CB[7]/MB nanohybrid compared with those of free $\mathrm{MB}$ and $\mathrm{CB}[7]$ (Fig. 8 and $\mathrm{S7}$ ). With the exception of the protons of the dimethylamino groups which underwent a slight low field shift, the other MB protons, mainly those close to the sulphur atom as well as those of the CB moiety, shifted to high field. Remarkably, the number of signals for the $\mathrm{MB}$ moiety of UCNP@CB[7]/MB was the same as that for MB, thus ruling out the coordination of MB through one of the dimethylamino groups. Consequently, we propose that $\mathrm{MB}$ stands symmetrically located on the $\mathrm{CB}[n]$ free carbonyl portal and it is bound to the carbonyls via the MB central sulphur cation (Fig. 2).

Similar experiments were carried out with pyronin Y (PYY). The UCNP emission was considerably quenched while using UCNP@CB[6] and UCNP@CB[7], but no emission changes were detected in the case of UCNP@CB[8] (Fig. 9).

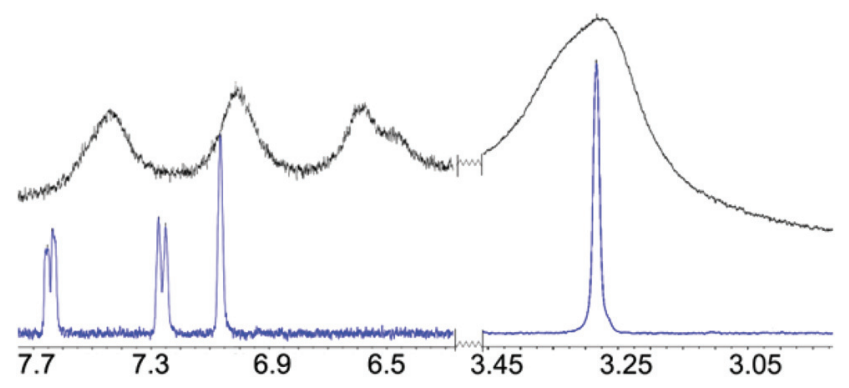

Fig. 8 Comparison between the ${ }^{1} \mathrm{H}-\mathrm{NMR}$ spectrum of UCNP@CB[7](aMB (top) and that of MB (bottom).
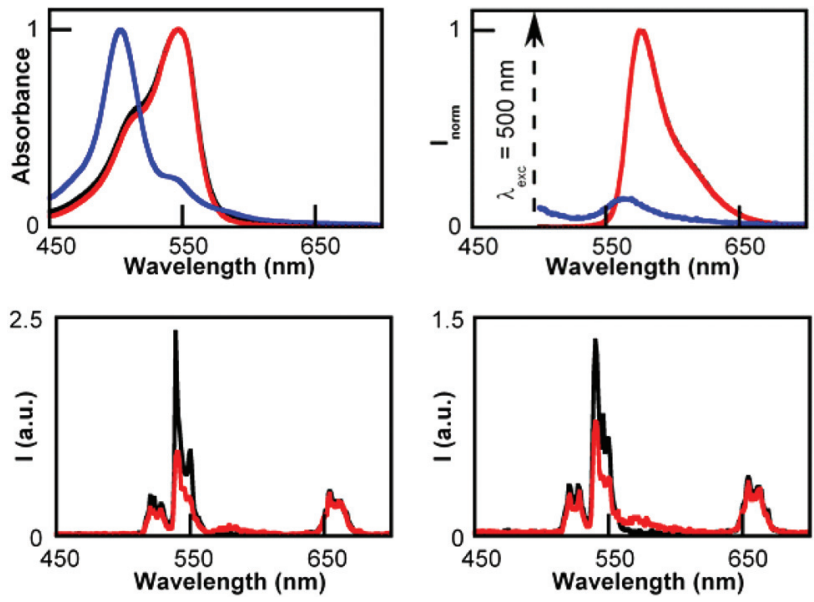

Fig. 9 Top: (left) Absorption and (right) emission spectra ( $\lambda_{\text {ex }} 540 \mathrm{~nm}$ ) of water dispersion of UCNP@CB[n]/PYY containing PYY and CB at the same concentration: UCNP@CB[6]/PYY (black line), UCNP@CB[7]/PYY (red line), and UCNPACB[8]/PYY (blue line); the absorption spectra of the UCNP@CB[n]/PYY mixtures as well as the emission spectra of UCNP@CB[n]/PYY ( $n=6,7$ and 8 ) have been normalized. Bottom: Emission spectra $\left(\lambda_{\text {ex }} 980 \mathrm{~nm}\right)$ of UCNPaCB $[n]$ ( $n=6$ and 7, at the left and right hand, respectively) before (black line) and after (red line) the addition of PYY; PYY and CB were at the same concentration. 
As observed for $\mathrm{MB}$, the different trend in the nanohybrid emission quenching was consistent with the formation of UCNP@CB[ $n] @ P Y Y(n=6$ and 7$)$ nanohybrids. The absorption spectra of the UCNP@CB $[n] / \mathrm{PYY}$ mixtures showed the typical band of the monomer at $\lambda_{\max } 545 \mathrm{~nm}$ for $n=6,7$, while the PYY dimer ( $\lambda_{\max } 500 \mathrm{~nm}$ ) was observed for $n=8$ (Fig. 9, top; Fig. S8 $\dagger$ ). In contrast to the MB emission, the PYY band slightly overlaps with that of the UCNP (Fig. 9, top). Consequently, the emission spectra at $\lambda_{\text {ex }} 545 \mathrm{~nm}$ of UCNP@CB[ $\left.n\right]$ (aPYY $(n=6,7)$ showed the PYY monomer emission (peak at $\lambda_{\max } 563 \mathrm{~nm}$ ), thus evidencing the energy transfer from the UCNP to PYY (Fig. 9, bottom; see the proposed interaction between UCNP@CB[7] and PYY in Fig. S9†).

\section{Recovery of the UCNP@CB nanohybrids}

UCNP@CB $[n] / \mathrm{MB}$ solutions containing the same concentration of $\mathrm{CB}$ and $\mathrm{MB}$ were centrifuged at $10000 \mathrm{rpm}$ for 10 minutes at room temperature. The absorption spectrum of the precipitate and the supernatant were compared with that of the corresponding UCNP@CB[n]/MB mixture (Fig. 10).

These spectra showed that in all cases, most of the $\mathrm{MB}$ remained in the supernatant and the precipitate consisted of $\mathrm{UCNP} @ \mathrm{CB}[n]$. The supernatant of the UCNP@CB $[n] / \mathrm{MB}$
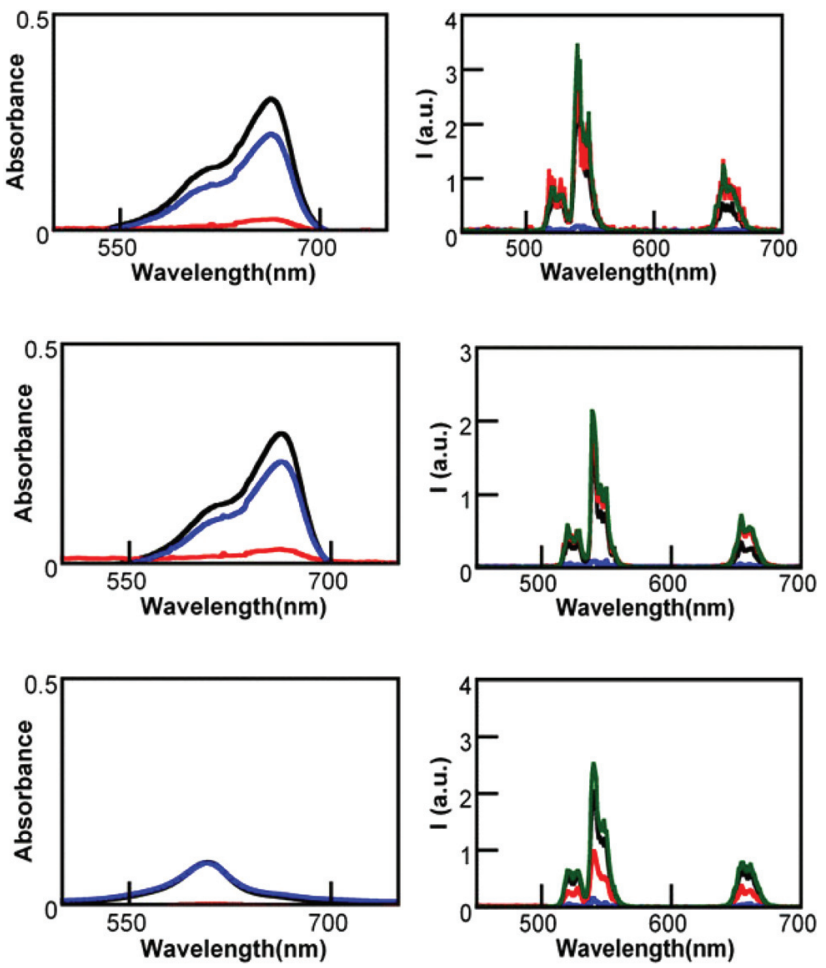

Fig. 10 Absorption (left) and emission spectra (right) at $\lambda_{\text {ex }}=980 \mathrm{~nm}$ of the UCNP@CB[n]/MB mixture (in black), the precipitate (in red) and the supernatant (in blue) obtained after centrifugation. Top: $n=6$; centre: $n=7$; bottom: $n=8$. For comparative purposes the emission spectrum of the corresponding UCNP@CB $[n]$ is included (in green). mixture $(n=6,7)$ contained only free $\mathrm{MB}$, while that of the UCNP@CB[8]/MB contained the $\mathrm{MB}_{2} @ \mathrm{CB}[8]$ inclusion complex. It has to be taken into account that the concentration of $\mathrm{CB}$ and $\mathrm{MB}$ in the UCNP@CB[8]/MB mixture was the same and, since $\mathrm{MB}$ was encapsulated as a dimer within the $\mathrm{CB}$ cavity, half of the $\mathrm{CB}$ units in UCNP@CB[8] remained empty. Therefore, the precipitate from the UCNP@CB[8]/MB mixture consisted of UCNPs partially capped with $\mathrm{CB}[8]$ units and, consequently, exhibited lower emission. These data suggest that in $\mathrm{UCNP@CB}[n] @ M B(n=6$ and 7$)$, the binding between $\mathrm{CB}$ and UCNP is stronger than that between $\mathrm{CB}$ and $\mathrm{MB}$. In the case of UCNP@CB[8]/MB, the binding of $\mathrm{MB}_{2}$ to the $\mathrm{CB}$ cavity proved to be stronger than the binding of $\mathrm{MB}_{2} @ \mathrm{CB}[8]$ to the UCNP surface.

\section{UCNP@CB@MB in singlet oxygen generation}

Methylene blue has been used in a variety of photochemical applications including photodynamic therapy. ${ }^{45,46}$ Therefore, the UCNP@CB[n]/MB mixtures with the higher molar concentration of $\mathrm{MB}$ were tested in the generation of singlet oxygen under near-IR irradiation (in particular, at $980 \mathrm{~nm}$ ). In principle, one would expect the UCNP@CB[8]/MB mixture to be the least efficient, since $\mathrm{MB}$ dimers are less efficient in the ${ }^{1} \mathrm{O}_{2}$ generation than the MB monomer. ${ }^{46}$

1,3-Diphenylisobenzofuran (DPBF) was used as a probe of single oxygen generation. This probe seemed to be adequate for these studies since it presents a broad absorption band in the 350-450 nm range where $\mathrm{MB}$ and $\mathrm{MB}_{2}$ exhibit negligible absorbance. DPBF is a good energy acceptor because it reacts rapidly with ${ }^{1} \mathrm{O}_{2}$. It does not react either with the ground state molecular oxygen or with the superoxide anion, and its only reaction with ${ }^{1} \mathrm{O}_{2}$ is chemical, leading to dibenzoylbenzene (DBB), which does not absorb in the 350-450 nm wavelength range.

Irradiation at $980 \mathrm{~nm}$ of $\mathrm{UCNP} @ \mathrm{CB}[n] @ \mathrm{MB}(n=6$ and 7$)$ nanohybrids in the presence of DPBF at different intervals of time (up to $70 \mathrm{~min}$ ) led to degradation of the probe, which was monitored by the loss of absorbance at $422 \mathrm{~nm}\left(A_{422}\right)$. Fig. 11 (top) shows the absorption spectra of the UC@CB[7]@MB solution in the presence of DPBF, before and after each irradiation, while Fig. 11 (bottom) shows the comparison between the loss of absorbance at $A_{422}$ in the presence of UCNP@CB[6] and of UCNP@CB[7]. This process involves several energy transfer processes: upconversion emission after excitation of $\mathrm{UCNP} @ \mathrm{CB}[n] @ \mathrm{MB}$ at $980 \mathrm{~nm}$; energy transfer from the UCNP to $\mathrm{MB}$ to lead eventually to the $\mathrm{MB}$ triplet excited state $\left({ }^{3} \mathrm{MB}\right)$ and energy transfer from ${ }^{3} \mathrm{MB}$ to molecular oxygen to form singlet oxygen, which reacts with DPBF to produce DBB. The rate of consumption of the substrate, calculated from the measurements at shorter irradiation time periods (30 seconds) for the first $15 \mathrm{~min}$ was $c a .80 \mathrm{~min}^{-1}$ and $11 \mathrm{~min}^{-1}$ for UCNP@CB[7] and UCNP@CB[6], respectively. 

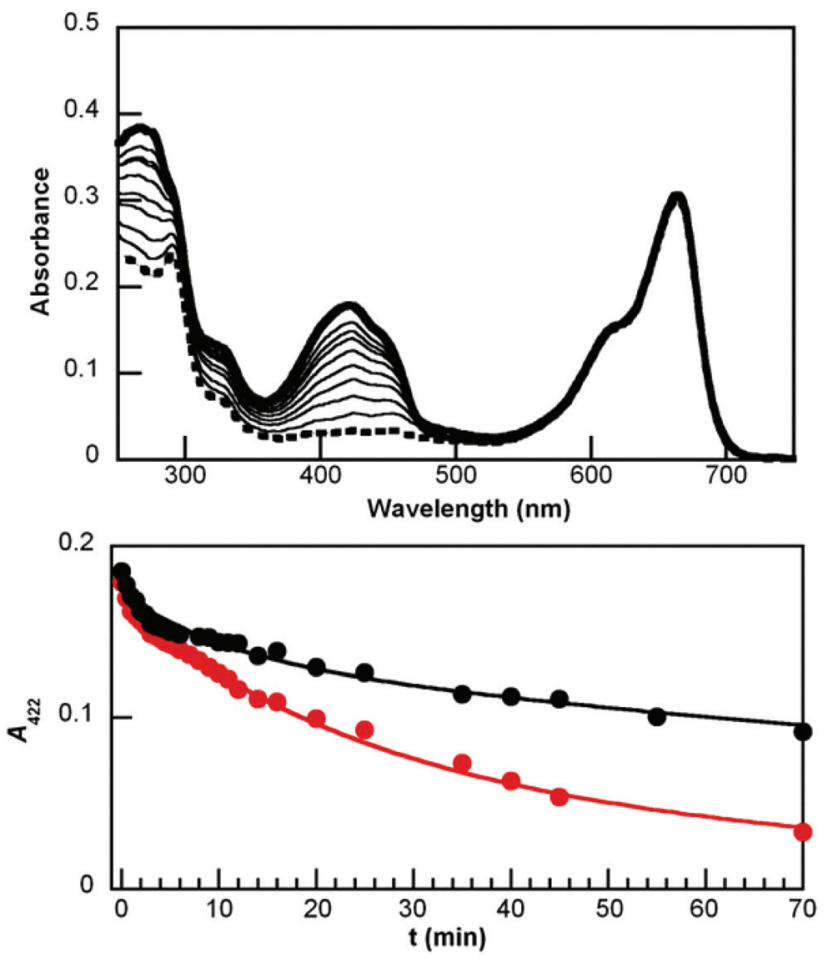

Fig. 11 (Top) Absorption spectrum of an aqueous dispersion of UCNP@CB[7] $A M B$ and DPBF (thick line) and its evolution after prolonged irradiation at $980 \mathrm{~nm}$ (up to $70 \mathrm{~min},--)$. (Bottom) Plot showing the degradation of DPBF (monitored at $422 \mathrm{~nm}$ ) in the presence of its corresponding UCNPaCB $[n] / \mathrm{MB}$ mixture: $n=6$ (black), $n=7$ (red).

The lower efficiency of the nanohybrid UCNP@CB[6]@MB than that of the UCNP@CB[7]@MB in halving the DPBF concentration is consistent with the lower luminescence of UCNP@CB[6]@MB (see Fig. 4) combined with the lower dye load of this nanohybrid (see TGA data).

\section{Conclusions}

We have demonstrated that the spontaneous adsorption of $\mathrm{CB}[n]\left(n=6,7\right.$, and 8) on $\mathrm{NaYF}_{4}: \mathrm{Er}^{3+}(2 \%), \mathrm{Yb}^{3+}(18 \%)$ nanoparticle surface gives rise to UCNP@CB $[n]$ nanohybrids, which have been proved to be highly stable. Those with $n=6$ and 7 lead to exclusion complexes with $\mathrm{MB}$, in which the interaction between $\mathrm{MB}$ and the $\mathrm{CB}$ carbonyl portal is weaker than that between the UCNP surface and CB. However, MB destabilised the interaction between the UCNP and the CB in UCNP@CB[8] due to the CB-encapsulation of the $\mathrm{MB}_{2}$ dimer. Similarly, PYY also led to spontaneous attachment to the $\mathrm{CB}$ free carbonyl portals of the UCNP@CB $[n](n=6,7)$ systems and energy transfer from the scaffold to PYY produced the emission of the dye under NIR-irradiation.

In addition, the UCNP@CB[7]@MB nanohybrid has been proved to be more efficient in the singlet oxygen generation than the UCNP@CB[6]@MB nanohybrid under NIR excitation. Therefore, UCNP@CB $[n]$ nanohybrids can act as scaffolds and efficient energy donors for cationic dyes by matching the nature of the UCNP with that of the dye. Taking into account the interest of tricyclic basic dyes in photodynamic therapy, these UCNP@CB[7]@dye supramolecular systems may be highly advantageous for this type of treatment.

\section{Acknowledgements}

We thank the Spanish Ministry of Economy and Competitiveness (Projects CTQ2011-27758; Ramón y Cajal contract for M.G.B. and F.P.U. grant for L.F.S.).

\section{Notes and references}

1 A. Gnach and A. Bednarkiewicz, Nano Today, 2012, 7, 532563.

2 F. Wang, D. Banerjee, Y. Liu and X. C. X. Liu, Analyst, 2010, 135, 1839-1854.

3 X. Wang, J. Zhuang, Q. Peng and Y. Li, Nature, 2005, 437, 121-124.

4 F. Wang and X. Liu, J. Am. Chem. Soc., 2008, 130, 56425643.

5 M. An, J. Cui, Q. He and L. Wang, J. Mater. Chem. B, 2013, 1, 1333-1339.

6 J.-C. G. Bunzli and S. V. Eliseeva, Chem. Sci., 2013, 4, 19391949.

7 L.-Q. Xiong, Z.-G. Chen, M.-X. Yu, F.-Y. Li, C. Liu and C.-H. Huang, Biomaterials, 2009, 30, 5592-5600.

8 J. Zhou, Y. Sun, X. Du, L. Xiong, H. Hu and F. Li, Biomaterials, 2010, 31, 3287-3295.

9 H. S. Qian, H. C. Guo, P. C.-L. Ho, R. Mahendran and Y. Zhang, Small, 2009, 5, 2285-2290.

10 J. C. Boyer, F. Vetrone, L. A. Cuccia and J. A. Capobianco, J. Am. Chem. Soc., 2006, 128, 7444-7445.

11 J.-C. Boyer, L. A. Cuccia and J. A. Capobianco, Nano Lett., 2007, 7, 847-852.

12 H.-Q. Wang and T. Nann, ACS Nano, 2009, 3, 3804-3808.

13 H.-X. Mai, Y.-W. Zhang, R. Si, Z.-G. Yan, L.-d. Sun, L.-P. You and C.-H. Yan, J. Am. Chem. Soc., 2006, 128, 6426-6436.

14 J. Chen and J. X. Zhao, Sensors, 2012, 12, 2414-2435.

15 M. Liras, M. González-Béjar, E. Peinado, L. FrancésSoriano, J. Pérez-Prieto, I. Quijada-Garrido and O. García, Chem. Mater., 2014, 26, 4014-4022.

16 V. Voliani, M. González-Béjar, V. Herranz-Pérez, M. Duran-Moreno, G. Signore, J. M. García-Verdugo and J. Pérez-Prieto, Chem. - Eur. J., 2013, 19, 13538-13546.

17 N. Bogdan, F. Vetrone, G. A. Ozin and J. A. Capobianco, Nano Lett., 2011, 11, 835-840.

18 Y. Ding, H. Zhu, X. Zhang, J. J. Zhu and C. Burda, Chem. Commun., 2013, 49, 7797-7799.

19 Q. Liu, C. Li, T. Yang, T. Yi and F. Li, Chem. Commun., 2010, 46, 5551-5553.

20 E. Masson, X. Ling, R. Joseph, L. Kyeremeh-Mensah and X. Lu, RSC Adv., 2012, 2, 1213-1247. 
21 G. Ghale and W. M. Nau, Acc. Chem. Res., 2014, 47, 21502159.

22 J. Kim, I. S. Jung, S. Y. Kim, E. Lee, J. K. Kang, S. Sakamoto, K. Yamaguchi and K. Kim, J. Am. Chem. Soc., 2000, 122, 540-541.

23 S. D. Choudhury, J. Mohanty, H. Pal and A. C. Bhasikuttan, J. Am. Chem. Soc., 2010, 132, 1395-1401.

24 H. Tang, D. Fuentealba, Y. H. Ko, N. Selvapalam, K. Kim and C. Bohne, J. Am. Chem. Soc., 2011, 133, 2062320633.

25 J. Lü, J.-X. Lin, M.-N. Cao and R. Cao, Coord. Chem. Rev., 2013, 257, 1334-1356.

26 Q. An, G. Li, C. Tao, Y. Li, Y. Wu and W. Zhang, Chem. Commun., 2008, 17, 1989-1991.

27 T. Premkumar, Y. Lee and K. E. Geckeler, Chem. - Eur. J., 2010, 16, 11563-11566.

28 X. Lu and E. Masson, Langmuir, 2011, 27, 3051-3058.

29 M. Cao, D. Wu, S. Gao and R. Cao, Chem. - Eur. J., 2012, 18, 12978-12985.

30 M. Cao, J. Lin, H. Yanga and R. Cao, Chem. Commun., 2010, 46, 5088-5090.

31 A. Lanterna, E. Pino, A. Doménech-Carbó, M. González-Béjar and J. Pérez-Prieto, Nanoscale, 2014, 6, 9550-9553.

32 R. W. Taylor, T.-C. Lee, O. A. Scherman, R. Esteban, J. Aizpurua, F. M. Huang, J. J. Baumberg and S. Mahajan, ACS Nano, 2011, 5, 3878-3887.

33 S. Kasera, F. Biedermann, J. J. Baumberg, O. A. Scherman and S. Mahajan, Nano Lett., 2012, 12, 5924-5928.

34 P. Thuéry, CrystEngComm, 2012, 14, 8128-8136.
35 L.-L. Liang, Y. Zhao, K. Chen, X. Xiao, J. Clegg, Y.-Q. Zhang, Z. Tao, S.-F. Xue, Q.-J. Zhu and G. Wei, Polymer, 2013, 5, 418-430.

36 O. A. Gerasko, E. A. Mainicheva, M. I. Naumova, O. P. Yurjeva, A. Alberola, C. Vicent, R. Llusar and V. P. Fedin, Eur. J. Inorg. Chem., 2008, 2008, 416-424.

37 K. Chen, L.-L. Liang, H.-J. Liu, Y.-Q. Zhang, S.-F. Xue, Z. Tao, X. Xiao, Q.-J. Zhu, L. F. Lindoy and G. Wei, CrystEngComm, 2012, 14, 7994.

38 L.-L. Liang, Y. Zhao, Y.-Q. Zhang, Z. Tao, S.-F. Xue, Q.-J. Zhu and J.-X. Liu, CrystEngComm, 2013, 15, 3943.

39 P. Montes-Navajas, A. Corma and H. Garcia, ChemPhysChem, 2008, 9, 713-720.

40 J. Lagona, P. Mukhopadhyay, S. Chakrabarti and L. Isaacs, Angew. Chem., Int. Ed., 2005, 44, 4844-4870.

41 P. F. C. Menezes, C. Bernal, H. Imasato, V. S. Bagnato and J. R. Perussi, Laser Phys., 2007, 17, 468-471.

42 K. Senthilkumar, P. Paul, C. Selvaraju and P. Natarajan, J. Phys. Chem. C, 2010, 114, 7085-7094.

43 Z. Li and Y. Zhang, Nanotechnology, 2008, 19, 345606-345610. 44 J. W. Lee, S. Samal, N. Selvapalam, H.-J. Kim and K. Kim, Acc. Chem. Res., 2003, 36, 621-630.

45 L. M. Moreira, J. P. Lyon, A. P. Romani, D. Severino, M. R. Rodrigues and H. P. M. de Oliveira, in Advanced Aspects of Spectroscopy, 2012, pp. 393-422.

46 J. P. Tardivo, A. Del Giglio, C. S. de Oliveira, D. S. Gabrielli, H. C. Junqueira, D. B. Tada, D. Severino, R. de Fátima Turchiello and M. S. Baptista, Photodiagn. Photodyn. Ther., 2005, 2, 175-191. 